\title{
ACE/SWICS OBSERVATIONS OF HEAVY ION DROPOUTS WITHIN THE SOLAR WIND
}

\author{
Micah J. Weberg ${ }^{1}$, Thomas H. ZurbuChen ${ }^{2}$, And Susan T. LePri ${ }^{3}$ \\ Department of Atmospheric, Oceanic, and Space Sciences, University of Michigan, Ann Arbor, MI, USA; \\ mjweberg@umich.edu, thomasz@umich.edu, slepri@umich.edu \\ Received 2012 February 2; accepted 2012 October 2; published 2012 October 31
}

\begin{abstract}
We present the first in situ observations of heavy ion dropouts within the slow solar wind, observed for select elements ranging from helium to iron. For iron, these dropouts manifest themselves as depletions of the $\mathrm{Fe} / \mathrm{H}$ ratio by factors up to $\sim 25$. The events often exhibit mass-dependent fractionation and are contained in slow, unsteady wind found within a few days from known stream interfaces. We propose that such dropouts are evidence of gravitational settling within large coronal loops, which later undergo interchange reconnection and become source regions of slow, unsteady wind. Previously, spectroscopic studies by Raymond et al. in 1997 (and later Feldman et al. in 1999) have yielded strong evidence for gravitational settling within these loops. However, their expected in situ signature plasma with heavy elements fractionated by mass was not observed prior to this study. Using data from the SWICS instrument on board the Advanced Composition Explorer ( $A C E$ ), we investigate the composition of the solar wind within these dropouts and explore long term trends over most of a solar cycle.
\end{abstract}

Key words: solar wind - Sun: abundances - Sun: corona - Sun: heliosphere

Online-only material: color figures

\section{INTRODUCTION}

One of the key discoveries of the Ulysses mission was the broad categorization of the solar wind into fast and slow types (Geiss et al. 1995a). Later studies (e.g., von Steiger et al. 2000) concluded that slow, unsteady wind compositional signatures are most similar to plasma in the magnetically closed corona (Zurbuchen 2007 and references therein). Fast wind on the other hand was associated with the open field regions called coronal holes (Nolte et al. 1976; Bame et al. 1993). According to the prevailing theories of slow wind origin and propagation (Antiochos 2011; Fisk 2003), the closed coronal loops must undergo some form of magnetic reconnection such that their otherwise trapped plasmas are released and can escape the Sun to become part of the solar wind. By studying the elemental abundances and charge state ratios of the solar wind we can thereby obtain direct information about processes occurring within the near-solar corona as well as those which occur during the propagation of solar wind within interplanetary space. This includes solar wind heating and acceleration processes, as well as processes which can change the relative elemental composition such as fractionation due to element-specific ionization or mass fractionation due to gravitational effects like gravitational settling.

Remote diagnostics of solar wind plasmas, such as by ultraviolet spectrometers on board the Solar and Heliospheric Observatory spacecraft (Kohl et al. 1995; Wilhelm et al. 1995), have also provided important information and constraints on heating, acceleration, and fractionation processes. One of the most important comparisons between in situ and remote observations is the average fractionation according to first ion-

\footnotetext{
1 PhD Candidate in Space and Planetary Physics, 2435 Space Research Building, 2455 Hayward Street, Ann Arbor, MI 48109-2143, USA.

2 Professor, Space Science and Aerospace Engineering, Associate Dean for Entrepreneurship, 2429 Space Research Building, 2455 Hayward Street, Ann Arbor, MI 48109-2143, USA.

3 Associate Research Scientist, 2417 Space Research Building, 2455

Hayward Street, Ann Arbor, MI 48109-2143, USA.
}

ization potential (FIP; von Steiger et al. 1997). In the closed corona, elements with low FIP (i.e., metals) are systematically enhanced over medium- and high-FIP elements when compared to their respective photospheric abundances. This same qualitative behavior, often called the "FIP-effect", is observed in the composition of the slow, unsteady wind. The slow wind is observed to be associated with coronal streamers and is in contrast to fast wind from coronal holes whose composition is observed, both in situ and remotely (Geiss et al. 1995a; Feldman 1998), to be nearly photospheric. This comparison creates a strong association between slow wind and the closed corona (i.e., coronal loops) which in turn implies the importance of some type of reconnection process responsible for the release of the plasma into the heliosphere (Fisk 2005 and references therein). It was further shown that the temporal structure of the slow solar wind coincides with geometric properties of the closed corona (Zurbuchen et al. 2000).

However, there has been a critical observation for which the above correlation between the closed corona and the compositional structure of slow wind has failed thus far. In 1997, Raymond et al. found evidence for gravitational settling within large, closed coronal loops in the equatorial streamer belt region. More specifically, they found that the total elemental abundances within the streamer center were lower than the abundances in the streamer edges, despite having nearly the same relative mixture of heavy elements. They suggested that the overall depletion of density within the streamer center was due to the plasma there being confined longer than the gravitational settling timescale. A similar study by Feldman et al. in 1999 also found compelling evidence for such a time-dependent settling process. They found that the line intensities of iron fell off faster with height than the lines of lighter elements. This mass-dependent fractionation is most easily explained by gravitational settling. Due to the often large geometric size of these coronal structures, one might expect that the gravitationally settled plasma should be observed in situ if the large, closed loops reconnect with the open field corona as has been theorized (Fisk 2003). This would lend further credence to the important connections between the closed 
corona and the slow, unsteady wind. Such an observational test is the key purpose of this paper.

The primary question we wish to address here is: if gravitational settling has been remotely observed in closed coronal loops and these loops are undergoing magnetic reconnection and releasing their plasma into the solar wind, do we observe mass-fractionated plasma in situ? Obviously, the preponderance of FIP-fractionated plasmas in the slow wind, observed in the average over many years (von Steiger \& Zurbuchen 2011), suggests that mass-fractionation processes cannot dominate the end solar wind abundances very often, else we would have already observed such plasmas using long-term averages and our above question would be redundant. Thus, it is assumed then that any mass-fractionated plasma that might exist within our data sets must be moderately short and hidden so as not to have been readily apparent. We must therefore first determine an appropriate indicator to aid us in searching for such events.

Based on its large mass and the expected fractionation patterns, it is clear that $\mathrm{Fe}$ is a very good indicator of gravitational settling, especially when contrasted with FIP fractionation. It is known that in FIP-fractionated plasmas Fe/O increases by factors of 2-4 (von Steiger et al. 2000). Conversely, in gravitationally settled plasmas we should see a substantial decrease of $\mathrm{Fe}$, even when compared to lighter elements that may be depleted themselves. That is, $\mathrm{Fe} / \mathrm{O}$ would be expected to decrease when compared to either slow or fast wind abundances and, additionally, should have the greatest relative depletion of all the elements.

\section{METHODOLOGY}

\subsection{Data Description}

We used $2 \mathrm{hr}$ averaged data from the Solar Wind Ion Composition Spectrometer (SWICS) on board the ACE satellite (Gloeckler et al. 1998). SWICS is a highly capable mass spectrometer with a time-of-flight telescope that has also flown on the Ulysses and Wind spacecraft (Gloeckler et al. 1992). SWICS is capable of measuring the speeds and densities of almost 40 different ions across the elements of $\mathrm{H}, \mathrm{He}, \mathrm{C}, \mathrm{N}$, $\mathrm{O}, \mathrm{Ne}, \mathrm{Mg}, \mathrm{Si}, \mathrm{S}$, and Fe. The accuracy of these measurements is generally within $20 \%$ or better, with some exceptions due to the limitations of counting statistics or the finite resolution of mass and charge which can lead to overlapping peaks of certain detected ions (see Appendix of von Steiger \& Zurbuchen 2010). For these reasons, $\mathrm{N}$ and $\mathrm{Ne}$ are not as well resolved as the other elements. In an effort to be as complete as possible while still maintaining proper quality and rigor, we have chosen to retain $\mathrm{N}$ within our analysis but will largely ignore Ne. Our analysis compares the heavy elements relative to $\mathrm{H}$, taking advantage of an extensive effort to cross-calibrate the $\mathrm{H}$ and $\mathrm{He}$ measurements of the two plasma sensors on $A C E$ : SWICS and SWEPAM (McComas et al. 1998). The crosscalibrated data are also available from $A C E$ level 3 data at http://www.srl.caltech.edu/ACE/.

\subsection{Selection Criteria}

We began by surveying the ratios of each SWICS-measured heavy element relative to hydrogen and searching for periods of time where the heavier elements, $\mathrm{Fe}$ in particular, seemed to drop significantly compared to the density variations of the rest of the solar wind. Specifically, we took the $\log _{10}$ of the $\mathrm{Fe} / \mathrm{H}$ ratio and computed its average and standard deviation within a given Carrington rotation (CR). We then identified all periods of time where the $\mathrm{Fe} / \mathrm{H}$ ratio dropped below one standard deviation from the $\mathrm{CR}$ average. We then required an event to have the following characteristics in order for us to consider it a possible heavy ion "dropout."

1. Duration $\geqslant 4 \mathrm{hr}$. This is mainly a result of the $2 \mathrm{hr}$ time resolution of our data set. While this limitation on length certainly prevents us from observing shorter events, which may still be relevant to our study, it should also filter out any possible short, unrelated transient events while leaving the longer, more interesting events intact. As we will discuss later in Section 4, most of the dropout events we have found are on the order of $15 \mathrm{hr}$ long or more and are thereby unaffected by the $4 \mathrm{hr}$ duration criterion. Furthermore, using the $2 \mathrm{hr}$ SWICS data allows for longer accumulation times which yields better differentiation between elemental species and insures that the data within our dropouts are of sufficient quality to run meaningful statistics.

2. One or more particularly low data points at least two standard deviations below the CR average. This criterion is, to a certain extent, arbitrary. However, we empirically determined that this was the simplest and most consistent test for picking out the cleaner, more significant events outside the normal variability of $\mathrm{Fe} / \mathrm{H}$.

\subsection{Event Filtering}

Using the method described above, we obtained a list of 315 possible dropout events within the time period of 2001-2009. This initial list was first filtered by rejecting 14 cases which had significant ( $>33 \%$ ) data gaps and then further refined by disregarding 15 events where the $\mathrm{X} / \mathrm{H}$ ratios changed primarily due to a rise in the hydrogen density while the elemental densities remained the same. We made this last determination by comparing the correlation coefficients between variations in $n_{\mathrm{H}} \& \mathrm{Fe} / \mathrm{H}$ and $n_{\mathrm{Fe}} \& \mathrm{Fe} / \mathrm{H}$. Those events where the drop in $\mathrm{Fe} / \mathrm{H}$ is due to a rise in hydrogen density will have a negative $\mathrm{Fe} / \mathrm{H}$ to $n_{\mathrm{H}}$ correlation coefficient of greater magnitude than the corresponding $\mathrm{Fe} / \mathrm{H}$ to $n_{\mathrm{Fe}}$ coefficient. Such "dropouts" do not represent actual depletions of heavy ions and are not the focus of our current analysis.

Additionally, especial care was taken to exclude from calculations and consideration any data that occur within interplanetary coronal mass ejections (ICMEs). ICMEs are transient events with their own set of compositional characteristics (Zurbuchen \& Richardson 2006) and we currently have no reason to associate them with processes such as gravitational settling which require a less chaotic environment. Richardson \& Cane (2010) have produced a fairly exhaustive list of ICMEs in the near-Earth environment using, in part, data from $A C E$. For our purposes, we simply exclude the time periods of the Richardson and Cane ICMEs from our calculations and ignore the 29 dropouts that begin or end within an ICME. Such periods may be an interesting topic for a future study.

Of the initial list of 315 candidate dropout events, 257 remained after filtering. These remaining dropouts all exhibit moderate-to-significant depletions of the heavy elements and we will constrain our subsequent statistics and discussion to only these events. First, in Section 3, we show an example of a particularly good dropout as a means to explain and illuminate our analysis method and primary conclusions. Then, in Section 4, we present statistics on fractionation patterns, occurrence rates, and average elemental abundances. Finally, we summarize our key observations and conclusions in Section 5. 

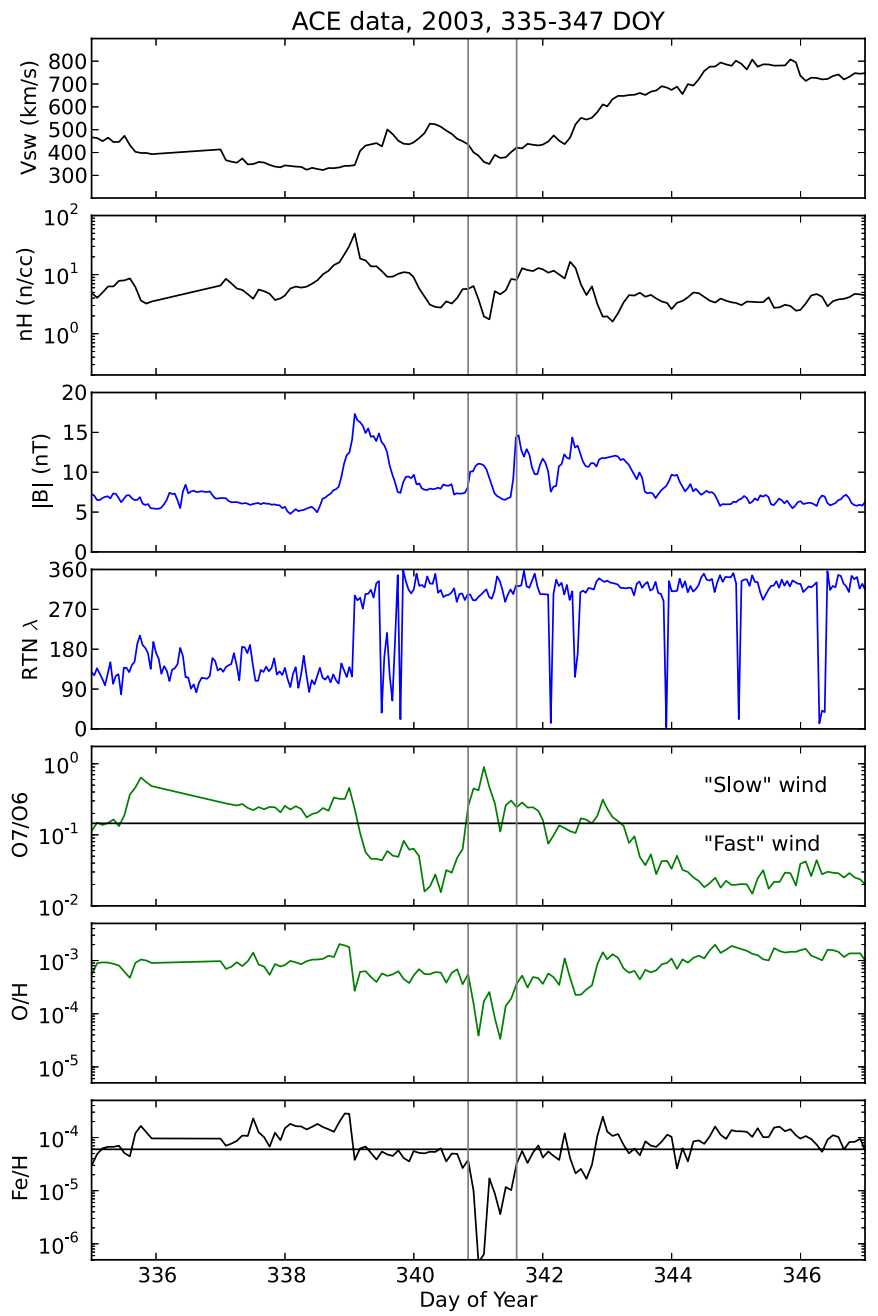

Figure 1. $A C E / S W I C S$ plasma overview for 335-347 DOY, 2003. The plots are, from top to bottom, solar wind speed, number density, magnetic field magnitude, RTN longitude, $\mathrm{O}^{7+} / \mathrm{O}^{6+}$ ratio, and the two elemental ratios $\mathrm{O} / \mathrm{H}$ and $\mathrm{Fe} / \mathrm{H}$. The solid horizontal line in the $\mathrm{Fe} / \mathrm{H}$ subplot shows the average ratio value within CR 2010 which contains the days plotted here. Also, the solid horizontal line in the $\mathrm{O}^{7+} / \mathrm{O}^{6+}$ subplot indicates the Zhao et al. (2009) solar wind type criteria of $\mathrm{O}^{7+} / \mathrm{O}^{6+}=0.145$. A heavy ion dropout was observed from 340.93 to 341.59 DOY where $\mathrm{Fe} / \mathrm{H}$ dropped by an order of magnitude. Two stream interfaces and their corresponding maxima of the magnetic field are visible at 339.2 and 341.59. The transition in $\lambda$ on 339 DOY indicates a heliospheric current sheet crossing. All plasma data are taken from the SWICS database and the magnetic field data are from the MAG instrument.

(A color version of this figure is available in the online journal.)

\section{EXAMPLE DROPOUT}

\subsection{Plasma Overview}

Figure 1 shows an overview of solar wind conditions in a $\sim 10$ day period of time centered on a dropout found in 2003 . The plots are, from top to bottom, solar wind speed $\left(V_{\mathrm{SW}}\right)$, density $\left(n_{\mathrm{H}}\right)$, magnetic field magnitude $|\mathbf{B}|$, RTN longitude $\lambda$, $\mathrm{O}^{7+} / \mathrm{O}^{6+}$ ratio (a good proxy for oxygen freeze-in temperature (Geiss et al 1995b)), and the two elemental ratios $\mathrm{O} / \mathrm{H}$ and $\mathrm{Fe} / \mathrm{H}$. There is a strong depletion in Fe that occurs for $\sim 16 \mathrm{hr}$ from 340.93 to 341.59 DOY (December 6th 22:13 to December 7th 14:17). Inside this period $\mathrm{Fe} / \mathrm{H}$ drops more than an order of magnitude, from an average of $9.37 \times 10^{-5}$ to $7.82 \times 10^{-6}$ (a total factor of $\sim 12$ ). A weaker depletion is also seen in the $\mathrm{O} / \mathrm{H}$ ratio $\left(9.53 \times 10^{-4}\right.$ to $1.34 \times 10^{-4}$ or a factor of 7.1$)$.
It has been established that one can differentiate between fast and slow solar wind using some combination of charge state ratios (Zhao et al. 2009; von Steiger et al. 2010). Using the $\mathrm{O}^{7+} / \mathrm{O}^{6+}$ criteria of Zhao et al. (as shown by the solid horizontal line in Figure 1) we note that the dropout is embedded in slow solar wind between two fast wind streams. Due to the differences in average velocity, fast wind streams often overtake slow wind streams and form stream interaction regions (SIRs). The boundary between the two streams within an SIR is called a stream interface (SI). Typical plasma characteristics of a slowto-fast wind SI include smoothly increasing solar wind velocity, a local maximum in tangential pressure, and a compression in both proton number density and magnitude of the interplanetary magnetic field (Jian et al. 2006). SIs, when mapped back to solar wind source regions on the Sun, lie near the separator of topologically open and closed magnetic fields in the corona (Wimmer-Schweingruber 1999). For the purposes of this study, we use a list of identified near-Earth SIRs, with SIs, produced by Jian et al. (2011) and available online at http://www-ssc. igpp.ucla.edu/ jlan/ACE/Level3/SIR_List_from_Lan_Jian.pdf. Our example dropout in Figure 1 comes right before a slow-tofast SI listed in the above Jian survey and visible in Figure 1 by the sharp jump in solar wind density at 341.59 DOY. Therefore, we deduce that our dropout originated from a closed, coronal loop similar to those that are thought to be the sources of the slow solar wind. We also note that a magnetic sector boundary is visible in Figure 1 on day 339 as an abrupt transition in $\lambda$. Such a transition normally indicates a crossing of the heliospheric current sheet (HCS).

\subsection{Elemental Fractionation}

We now define a "reduction factor" for each element by taking the average $\mathrm{X} / \mathrm{H}$ ratios 5 days before and after the dropout and dividing them by their corresponding averages within the dropout period. These reduction factors represent the depletions of each element relative to their abundances in the immediately neighboring plasma. Elements with larger reduction factors are therefore more depleted relative to $\mathrm{H}$ than those with smaller factors. If another dropout occurred within the same \pm 5 day window, it was omitted from the calculations of the averages since we desired to only compare to the unperturbed, background solar wind abundances. The \pm 5 day window allows for enough statistical significance in our analysis and exceeds the typical correlation timescales of compositional data (Zurbuchen et al. 1999, 2000). However, our analysis does not strongly depend on the actual duration of this interval. Figure 2 is a plot of the reduction factors computed for all SWICS-measured elements over the same time period as shown in Figure 1. The statistical uncertainty was determined using standard error propagation and from the standard error of the means (SEM) inside and outside the dropout. The left-hand side of the plot orders the elements by mass while the righthand side orders them by ionization potential, FIP. We also include $\mathrm{N}$ data, despite the inherent measurement challenges previously mentioned. The inclusion of this less certain element, however, does not significantly alter either our calculations or conclusions.

Figure 2 shows a clear rising trend in reduction factors for the heavier mass elements. This is in qualitative agreement with the fractionation signature expected from gravitational settling. While the right-hand side of Figure 2 may at first seem reminiscent of the standard FIP effect plots, with the low FIP elements having the largest values (see Figure 9 of von Steiger 
X/H Reduction Factors inside Dropout Event, 2003, 335-347 DOY

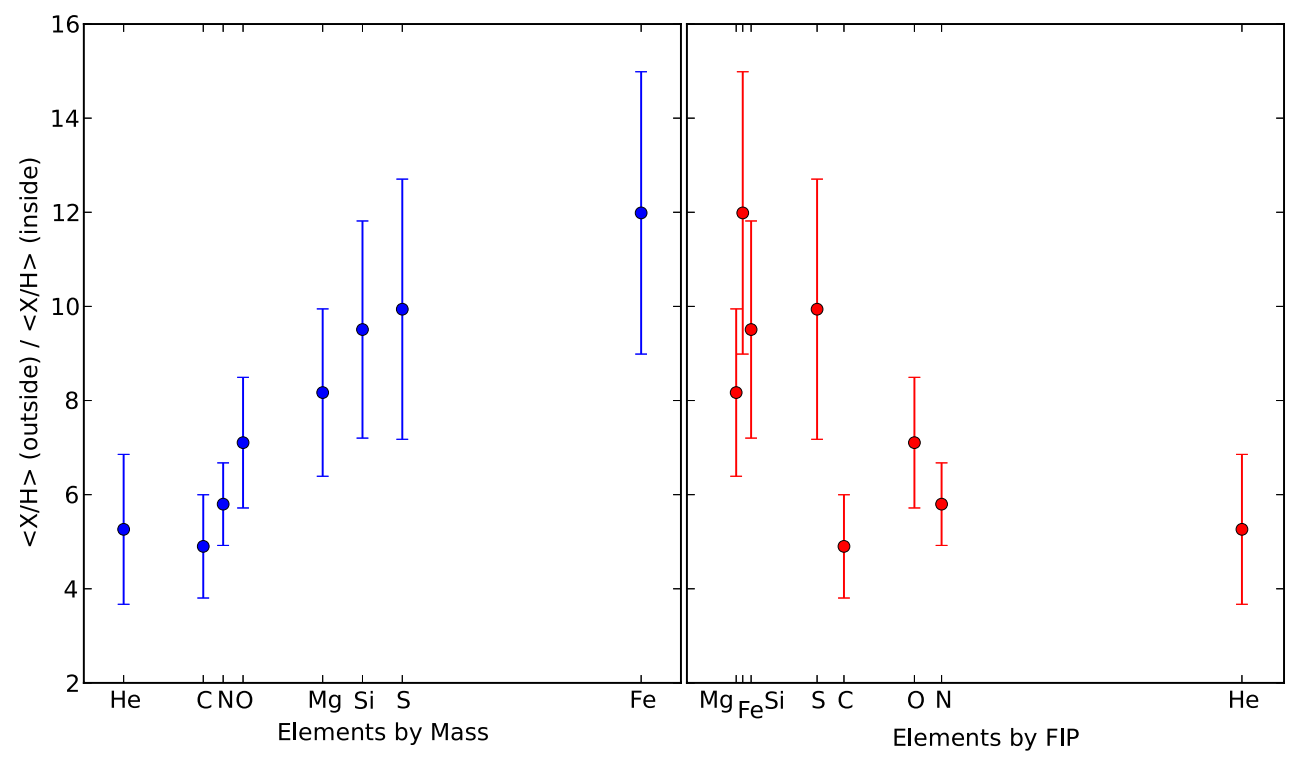

Figure 2. $\mathrm{X} / \mathrm{H}$ reduction factors inside the 335-347 DOY, 2003 dropout event shown in Figure 1. Reduction factors are computed by taking the average ratio 5 days before and after the dropout and dividing them by the ratios inside. Error bars were determined using the standard error of the means. Elements with larger reduction factors are more significantly depleted. The rising trend in reduction factors is in qualitative agreement with gravitational settling.

(A color version of this figure is available in the online journal.)

Comparison of X/H Ratios for Dropout Event, 2003, 335-347 DOY

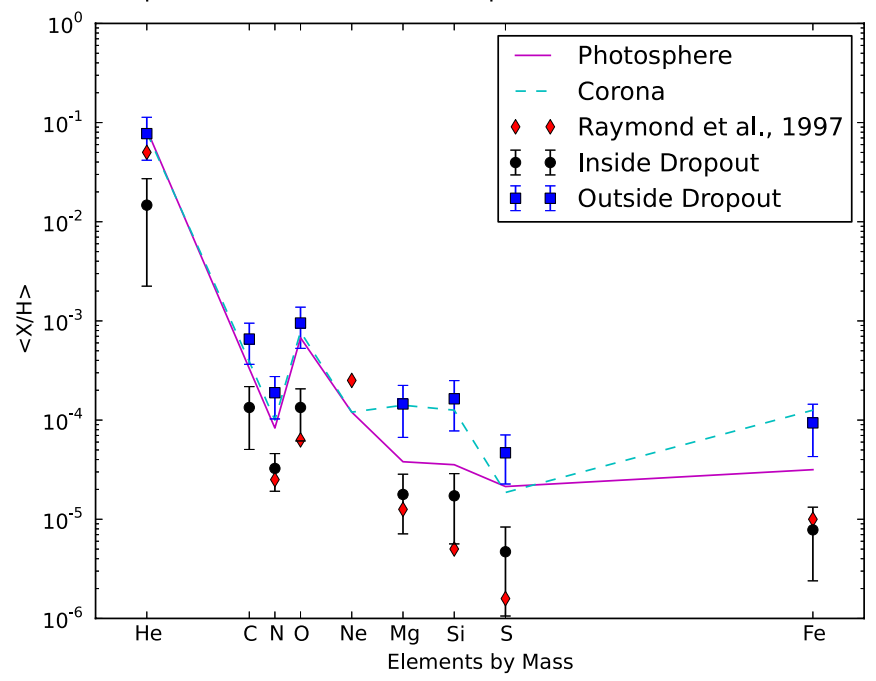

Figure 3. Comparison of $\mathrm{X} / \mathrm{H}$ ratios inside the 335-347 DOY, 2003 dropout event (black circles) to ratios within the streamer core observed by Raymond et al. (1997, red diamonds). Also shown are the ratios \pm 5 days outside the dropout (blue squares), in the photosphere (magenta line; Grevesse and Sauval 1998), and the corona (dashed, cyan line; Feldman et al. 1992). The observed dropout ratios are most similar to the streamer core.

(A color version of this figure is available in the online journal.)

et al. 1997), the values in our figure indicate depletions rather than enhancements and as such represent the opposite of what is expected in FIP-fractionated wind. There is no apparent ordering of the reduction factors according to FIP. The low FIP elements also happen to be heavier and will have large reduction factors anytime there is mass-dependent fractionation.

\subsection{Comparison to UVCS}

Figure 3 compares the $\mathrm{X} / \mathrm{H}$ ratios inside our dropout to those Raymond et al. (1997) remotely observed inside the center of an equatorial streamer. While it is important to note here that our example dropout and the streamer of Raymond et al. are separated by 7 years, there is still some use in making the comparison. The black circles in Figure 3 represent the ratios within our dropout while the red diamonds are the ratios computed from the elemental abundances reported in Table VII of the Raymond et al. paper. Also shown are the ratios outside the dropout (blue squares) along with typical photospheric (Grevesse \& Sauval 1998) and coronal (Feldman et al. 1992) values (magenta and dashed, cyan lines respectively). We can see that the ratios within our dropout compare favorably, within the error bars, with those of the streamer center. Error bars are not shown for the UVCS measurements since uncertainty and error ranges were not published within the Raymond et al. paper. We also cannot say much about carbon since we lack a streamer abundance to compare with. As mentioned in Section 1, Raymond et al. argue that the abundances in the streamer center are partially due to gravitational settling. This would seem to lend further credence to our analysis of the mass-dependent fractionation seen in Figure 2. However, we must be careful not to read too much into the apparent agreement in Figure 3 since, as stated earlier, our dropout and the streamer of Raymond et al. represent observations from two different parts of the solar cycle. The primary point of Figure 3 is to show that the plasma within our example dropout is entirely unlike typical coronal or photospheric plasmas which have been shown to be similar to slow and fast solar wind, respectively (von Steiger et al. 1997, 2000; Zurbuchen et al. 2002). Instead, the plasma within our dropout is most similar to that observed within a streamer center which was thought to be magnetically closed, thereby suggesting that the plasma within our dropout originated from a similar structure.

\section{SURVEY STATISTICS}

\subsection{Quantifying “Mass Fractionation”}

In our analysis, we used a simple mathematical test to determine if a given dropout appears to be mass fractionated 


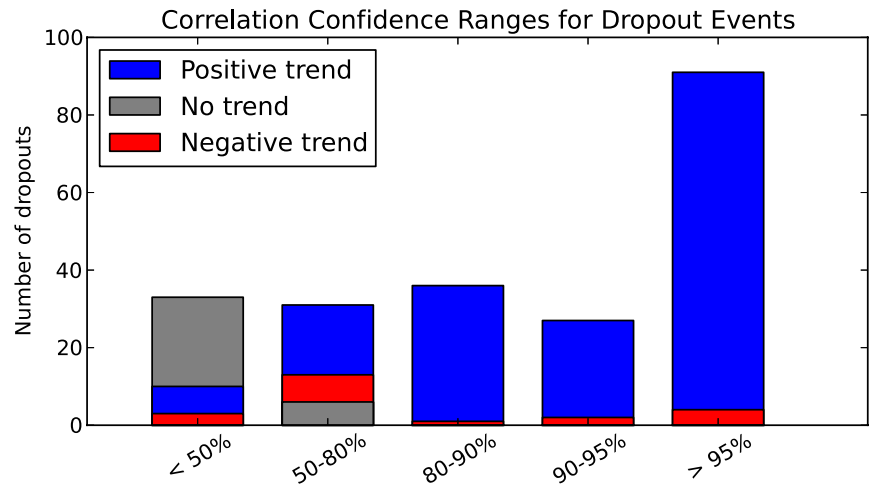

Figure 4. Distribution of dropout events into common confidence ranges. The higher the confidence levels, the stronger the correlation between element mass and relative reduction factor. We define only the 91 positive trend events in the $\geqslant 95 \%$ range as "mass fractionated."

(A color version of this figure is available in the online journal.)

or not. We began by fitting a series of linear equations to the reduction factors of each dropout. In each iteration of the series, we omitted one of the elements from the fit (this is known as the "Jackknife" method) and thereby obtained a distribution of slopes reflecting the general trend in the reduction factors. In all of the fits, we ignored the reduction factor for He for two reasons: (1) given its considerably higher density compared to the minor ions, He is dynamically important and most properly considered as part of the main ion population (Bodmer \& Bochsler 2000); and (2) He is known to be depleted near the HCS (Borrini et al. 1981; Suess et al. 2009). Both factors could lead to anomalously large reductions in He which could interfere with our process to determine the overall trend in reduction factors. Finally, we computed the correlation coefficient, $r$, for each average fitted slope. According to standard statistical tables (David 1938), the $95 \%$ significance level for 5 degrees of freedom ( 7 data points, one per element, minus 2 variables) is denoted by $|r| \geqslant 0.754$.

Of the 257 dropouts identified in the period 2001-2009, 195 $(75.9 \%)$ have a general upward trend where the average minus one standard deviation of the fitted slopes is positive. However, only 91 (35.4\% of all events) have a strong correlation $(|r| \geqslant$ 0.754 ) between element mass and reduction factor. It is only this subset of 91 events that we define as "mass fractionated". As for the remaining 166 non-mass-fractionated events, 104 $(40.5 \%)$ have positive trends with moderate or weak correlation, $23(8.9 \%)$ have negative trends (4 with strong correlation, 19 with moderate or weak), and $39(15.2 \%)$ have no clear trend up or down (note that percentages are relative to the entire set of 257 dropouts). Figure 4 shows the distribution of events into common confidence ranges. Higher confidence levels correspond to a stronger correlation between element mass and relative reduction factor.

\subsection{Occurrence Rates over Most of a Solar Cycle}

Figure 5 is a bar graph depicting the yearly distribution of all possible dropouts with filled regions indicating the portion of them which are mass fractionated according to the method of Section 4.1. It is interesting to note that while there seem to be more dropouts later in the solar cycle near the 2008 minimum, the number of mass-fractionated cases does not vary proportionately. It is perhaps worth noting that some of our dropouts were observed during the recent anomalous minimum, which exhibited a $3 \%$ reduction in solar wind speed, $17 \%$ reduction in density, and $14 \%$ reduction in temperature when compared to previous minima (McComas et al. 2008). It is entirely possible that some, but not necessarily all, of the other dropouts originally had mass-fractionated plasma but the abundance signatures were later erased by other processes, such as those described by Schwadron et al. (1999).

\subsection{Average Values}

The average duration of all possible dropouts is $17.56 \pm$ $12.75 \mathrm{hr}$ and the mass-fractionated cases are similar with an average of $16.33 \pm 10.17 \mathrm{hr}$. Within the mass-fractionated cases, iron is depleted by factors ranging from 2.12 to 27.69 with an average of $7.42 \pm 5.04$. Table 1 shows the average elemental ratios relative to hydrogen for the 91 mass-fractionated dropouts. For comparison, we have computed the average ratios within typical slow solar wind (von Steiger et al. 2000, 2010), the photosphere (Grevesse \& Sauval 1998), and the solar corona (Feldman et al. 1992). The uncertainties listed for our results were computed using the standard error of the means. The values shown in parentheses represent the reduction factors of the massfractionated dropouts relative to the given reference ratios (i.e., $\left.\left[\mathrm{X}_{\mathrm{Ref}} / \mathrm{H}_{\mathrm{Ref}}\right] /\left[\mathrm{X}_{\mathrm{MF}} / \mathrm{H}_{\mathrm{MF}}\right]\right)$.

Table 1 provides some qualitative details concerning the relative importance of fractionation processes in the dropout events without needing to resort to a complete theoretical or mathematical model. When compared to typical slow wind or coronal $\mathrm{X} / \mathrm{H}$ ratios, the mass-fractionated dropouts show greater depletions for heavier elements, with Fe being the most depleted. This is as to be expected of a mass-dependent process such as gravitational settling and further confirms that the apparent ordering according to mass in the "massfractionated" dropouts is a statistically important effect and not some artifact caused by the averaging done in our analysis. The fractionation pattern when comparing the dropouts to the photosphere is more complicated. We can nevertheless still glean one useful conclusion from the comparison. Most fractionation processes, such as FIP-fractionation, are defined relative to photospheric abundances and are caused by processes that occur in the chromosphere or corona (Geiss 1982; Meyer 1991). If gravitational settling was considerably stronger than all other fractionation processes inside the mass-fractionated dropouts, we would expect to see a moderate or at least weak correlation with mass when compared to the photospheric abundances. However, as can be seen in the reduction factors of Column 3 of Table 1, this is clearly not the case. Therefore gravitational settling can, at most, only be moderately stronger than other fractionation processes rather than overwhelmingly dominant. The exact mixture of processes responsible for the mass-fractionated dropouts is, of course, beyond the limited insight afforded by our analysis of data trends and is a question that can perhaps be best answered within the framework of a full mathematical model.

\subsection{Comparison of Mass-fractionated Dropouts to Other Plasmas}

Similar to Figure 3, Figure 6 depicts the elemental ratios from Table 1 in graphical form. Of particular interest is the comparison between the average $\mathrm{X} / \mathrm{H}$ ratios of all 91 massfractionated dropouts (black circles) and the $\mathrm{X} / \mathrm{H}$ ratios inside the streamer center observed by Raymond et al. (red diamonds). We have also plotted the median dropout $\mathrm{X} / \mathrm{H}$ ratios (black line). As can be seen in Figure 6, the average and median dropout X/H ratios tend to be a bit higher than the streamer center but are still 


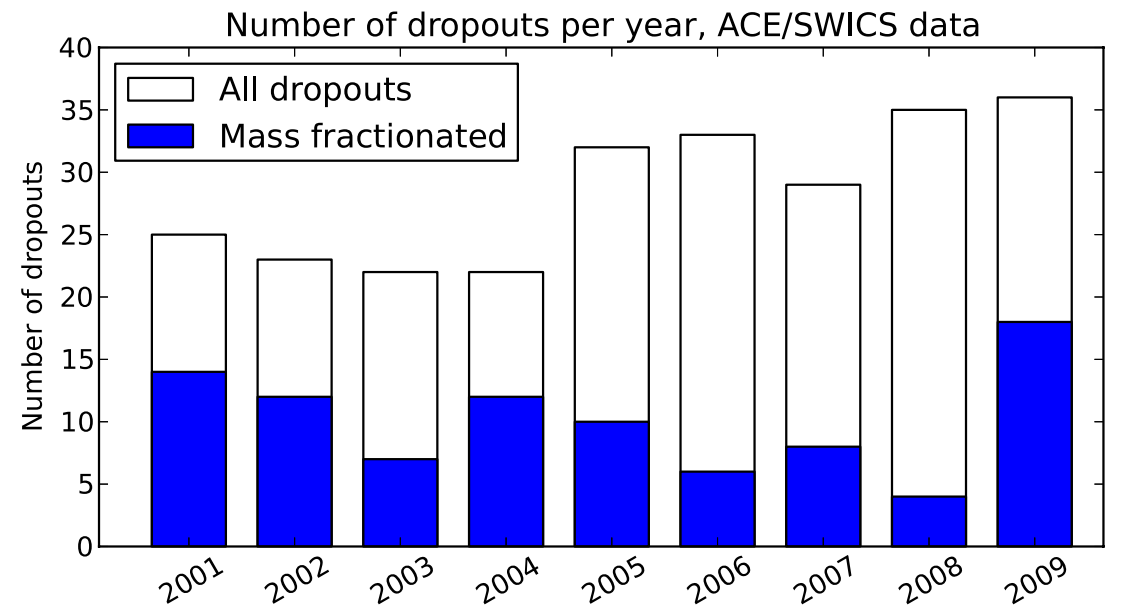

Figure 5. Number of potential dropouts per year. Shaded regions indicate mass-fractionated cases. There are more total dropouts near the 2008 solar minimum, however, the mass-fractionated cases do not seem to have any solar cycle dependence.

(A color version of this figure is available in the online journal.)

Table 1

Comparison of Average Ratios Relative to Hydrogen

\begin{tabular}{lcccc}
\hline \hline Ratio & $\begin{array}{c}\text { Mass-fractionated } \\
\text { Dropouts }\end{array}$ & $\begin{array}{c}\text { Typical Slow } \\
\text { Solar Wind }\end{array}$ & Photosphere $^{\mathrm{b}}$ & Corona $^{\mathrm{c}}$ \\
\hline $\mathrm{He} / \mathrm{H}$ & $1.14 \pm 0.15 \times 10^{-2}$ & $3.91 \times 10^{-2}(3.43)$ & $8.51 \times 10^{-2}(7.46)$ & $7.94 \times 10^{-2}(6.96)$ \\
$\mathrm{C} / \mathrm{H}$ & $9.76 \pm 0.97 \times 10^{-5}$ & $2.91 \times 10^{-4}(2.98)$ & $3.31 \times 10^{-4}(3.39)$ & $3.89 \times 10^{-4}(3.99)$ \\
$\mathrm{N} / \mathrm{H}$ & $2.41 \pm 0.20 \times 10^{-5}$ & $3.41 \times 10^{-5}(1.41)$ & $8.32 \times 10^{-5}(3.45)$ & $1.00 \times 10^{-4}(4.15)$ \\
$\mathrm{O} / \mathrm{H}$ & $1.10 \pm 0.12 \times 10^{-4}$ & $4.35 \times 10^{-4}(3.95)$ & $6.76 \times 10^{-4}(6.15)$ & $7.76 \times 10^{-4}(7.05)$ \\
$\mathrm{Mg} / \mathrm{H}$ & $1.81 \pm 0.19 \times 10^{-5}$ & $6.30 \times 10^{-5}(3.48)$ & $3.80 \times 10^{-5}(2.09)$ & $1.41 \times 10^{-4}(7.79)$ \\
$\mathrm{Si} / \mathrm{H}$ & $1.56 \pm 0.17 \times 10^{-5}$ & $6.50 \times 10^{-5}(4.17)$ & $3.55 \times 10^{-5}(2.28)$ & $1.26 \times 10^{-4}(8.08)$ \\
$\mathrm{S} / \mathrm{H}$ & $4.92 \pm 0.48 \times 10^{-6}$ & $2.17 \times 10^{-5}(4.41)$ & $2.14 \times 10^{-5}(4.35)$ & $1.86 \times 10^{-5}(3.78)$ \\
$\mathrm{Fe} / \mathrm{H}$ & $7.20 \pm 0.74 \times 10^{-6}$ & $4.91 \times 10^{-5}(6.82)$ & $3.16 \times 10^{-5}(4.39)$ & $1.26 \times 10^{-4}(17.5)$ \\
\hline
\end{tabular}

Notes.

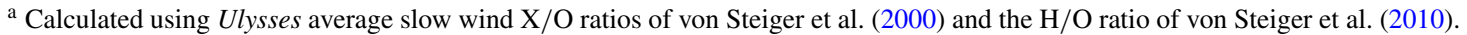

${ }^{\mathrm{b}}$ From Grevesse \& Sauval (1998).

${ }^{\mathrm{c}}$ From Feldman et al. (1992).

much lower than the photospheric, coronal, and slow wind ratios (magenta, cyan, and blue lines, respectively). This suggests that plasmas with heavy ion dropouts are, as a whole, most similar to observations of the streamer center, which Raymond et al. argued must be a magnetically closed region that has been, at least partially, depleted by gravitational settling. Note, however, that Figure 6 compares an ensemble of dropouts (spanning the years 2001-2009) with a single specific streamer observed in 1996 July and the quantitative details of this comparison should not be overinterpreted.

\subsection{Relation to Stream Interfaces}

Among the 91 mass-fractionated dropouts, 44 (48.4\%) occur either within stream interaction regions on the Jian et al. SIR list (23 cases) or just outside of an SIR within 2 days (21 cases). Such a low correlation is not entirely surprising because massfractionated dropouts observed in situ are expected to map to the edges of coronal holes. SIRs on Jian's list form when a stream of slow-velocity wind is overtaken by a faster stream. Therefore, plasmas near these SIs represent only the preceding edges of coronal holes. Since gravitational settling may occur within closed loops on either side of a coronal hole, we should not expect much more than half of all mass-fractionated dropouts to occur near the preceding edge, stream interfaces. Additionally, we can rule out the possibility that these dropouts are caused by some kind of process or interaction inside the compressive region of slow-to-fast SIR since in those events we would expect to see a clear preference for the leading edge SI.

This lack of preference persists when we consider all of the dropouts and not just the mass-fractionated ones. Of the 257 possible dropouts, $158(61.5 \%)$ are found near SIs. This amounts to $\sim 44 \%$ of the 359 SIRs on the Jian list between the years 2001 and 2009. Again, as with the mass-fractionated cases, the distribution of dropouts is split almost evenly between events occurring inside SIRs (86 cases) and just outside SIRs within 2 days ( 72 cases). Figure 7 is a histogram of the time between all 158 correlated dropouts and the nearest SI. Negative numbers indicate dropouts that come before the SI and positive numbers denote those that come after. The peak of the distribution is less than a day before the SIs, a period most closely tied to slow solar wind. The average of the absolute time offsets is 0.5137 days for events occurring inside SIR, 1.1341 days for events occurring near but outside SIR, and 0.7964 for all events together. It is difficult to say whether the remaining 99 uncorrelated dropouts have any relation to the trailing edges of coronal holes. This is because the fast stream outruns the slow stream that follows and creates a sort of rarefaction with no clear boundaries and a 
Comparison of X/H Ratios for All Mass Frac. Dropouts, 2001 - 2009

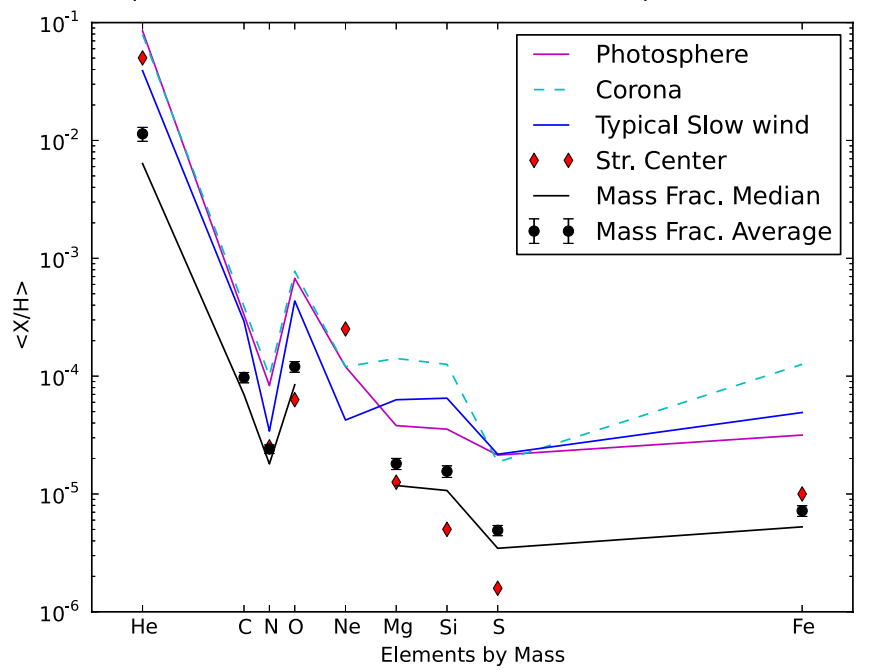

Figure 6. Average $\mathrm{X} / \mathrm{H}$ ratios within all 91 mass-fractionated dropouts (black dots) compared to the $\mathrm{X} / \mathrm{H}$ ratios inside the streamer center observed by Raymond et al. (1997; red diamonds). Error bars on the dropout values represent the standard error of the means as found in the first column of Table 1. Also plotted are the median dropout values (black line) and typical photospheric (magenta line; Grevesse \& Sauval 1998), coronal (cyan line; Feldman et al. 1992), and slow solar wind ratios (blue line; von Steiger et al. 2000, 2010). Like in Figure 3, the average dropout ratios are most similar to those in the streamer core.

(A color version of this figure is available in the online journal.)

fair bit of mixing. Further research, perhaps using the variability of charge state ratios to differentiate between streams, should hopefully yield some proper statistics. In particular, it will be interesting to see if the smaller peaks in Figure 6 between 1 and 2 days after the SI have any relation to the trailing edges of particularly narrow coronal holes.

\section{SUMMARY AND CONCLUSIONS}

We have shown here $A C E / S W I C S$ observations of heavy ion dropouts within the solar wind. These observations represent the first in situ measurements of mass-fractionated solar wind and as such lend further credence to models that associate slow, unsteady wind with plasma from coronal loops.

Key observations include the following.

1. Most dropouts are found embedded within slow solar wind plasma with about half of them lying less than 1.5 days from interfaces between slow and fast streams. These SIs correspond to the boundaries between open and closed magnetic fields in the solar corona, where previous studies (Raymond et al. 1997; Feldman et al. 1999) found strong evidence for gravitational settling. The correspondence between SIs and dropout periods is important because it suggests that those dropouts found right before an SI originated from a coronal loop on the edge of a coronal hole. Additionally, the given occurrence rate does not indicate any preference for the leading or trailing edge of a coronal hole and rules out the possibility that the dropouts are caused by interactions within the SIs themselves.

2. Heavier ions generally exhibit larger depletions, with $35.4 \%$ of all possible dropouts demonstrating mass-dependent fractionation. This is consistent with what we should expect of gravitational settling. Within the mass-dependent cases, iron was found to be depleted by factors ranging

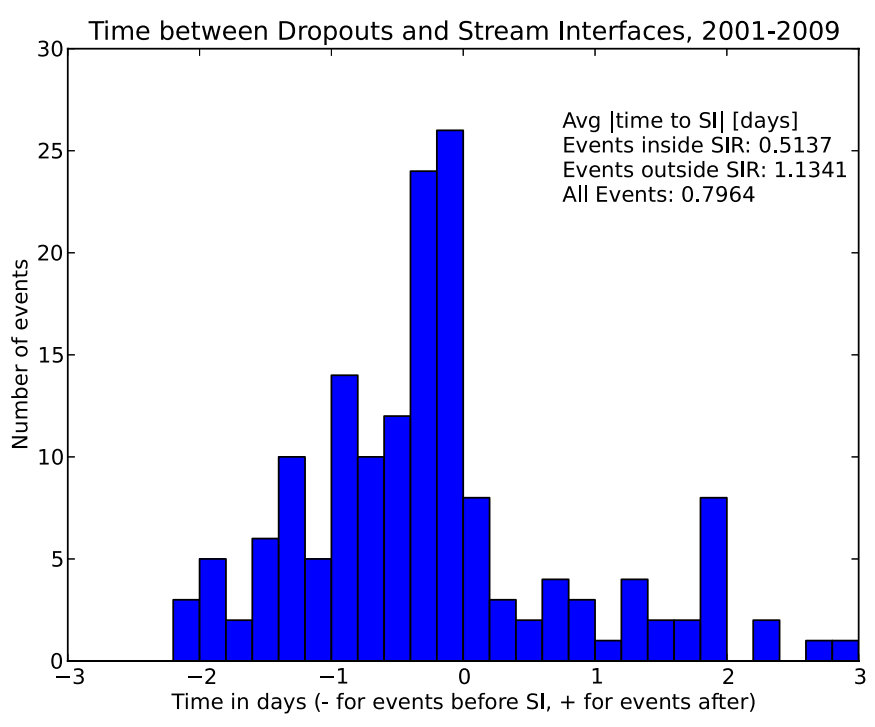

Figure 7. Time in days between dropout centers and nearby stream interfaces (SIs) for the 159 events, out of all 257 possible dropouts, which are reasonably close to SIs. Negative (positive) numbers indicate dropouts that come before (after) the SI. Most of the dropouts are found right before the SI, during periods of slow solar wind.

(A color version of this figure is available in the online journal.)

from 2.12 to 27.69 with an average of 7.42 . Some of these cases compare favorably with the aforementioned remote observations, giving us added confidence that the fractionation in those events is, at least partially, due to gravitational settling.

3. The yearly number of mass-fractionated dropouts does not appear to have a strong solar cycle dependence. However, there are more total dropouts found near solar minimum. It is entirely possible, but not yet determined, that some of the non-mass-fractionated cases represent plasmas which were initially depleted by gravitational settling but the mass dependence was partially scrambled and hidden by other coronal fractionation processes.

One quite reasonable question to ask is why these dropouts have not been previously reported. There are two main reasons for this. First of all, the dropouts discussed in this paper are on the order of less than a day. Therefore any study looking at long-term variations using averages over more than a day, or in some cases even just half a day, would not have been able to find the depletions. Second, most previous studies of heavy ion abundances have used the $\mathrm{X} / \mathrm{O}$ elemental ratios rather than the $\mathrm{X} / \mathrm{H}$ ratios we used here. This was because properly cross-calibrated $\mathrm{H}$ densities were not available from the SWICS instrument until recent years. Since both $\mathrm{O}$ and $\mathrm{Fe}$ are depleted in the observed dropouts, the corresponding dips in the $\mathrm{Fe} / \mathrm{O}$ would be smaller and more easily overlooked.

The in situ observations of solar wind elemental abundances are an important piece of solar and heliospheric research. Massfractionated plasmas linked to gravitational settling can provide unique insight into the subtle interplay between coronal processes. Additionally, mass fractionation can be used as a signature to connect in situ observations to elemental abundances determined by remote spectroscopy. Dropouts such as those presented here also yield clues about the source regions of solar wind and could be used as an additional test of slow wind origin theories. 
This work was supported, in part, by grants from ACE (44 A-1085637), Living with a Star (NNX10AQ61G), and NASA contract NNX08AM64G.

\section{REFERENCES}

Antiochos, S. K., Mikić, Z., Titov, V. S., Lionello, R., \& Linker, J. A. 2011, ApJ, 731,112

Bame, S. J., Goldstein, B. E., Gosling, J. T., et al. 1993, Geophys. Res. Lett., 20,2323

Bodmer, R., \& Bochsler, P. 2000, J. Geophys. Res., 105, 47

Borrini, G., Wilcox, J. M., Gosling, J. T., Bame, S. J., \& Feldman, W. C. 1981, J. Geophys. Res., 86, 4565

David, F. N. 1938, Tables of the Correlation Coefficient (London: Biometrika)

Feldman, U. 1998, Space Sci. Rev., 85, 227

Feldman, U., Doschek, G. A., Schühle, U., \& Wilhelm, K. 1999, ApJ, 518, 500

Feldman, U., Mandelbaum, P., Seely, J. F., Doschek, G. A., \& Gursky, H. 1992, ApJS, 81, 387

Fisk, L. 2003, J. Geophys. Res., 108, 1157

Fisk, L. 2005, ApJ, 626, 563

Geiss, J. 1982, Space Sci. Rev., 33, 201

Geiss, J., Gloeckler, G., \& von Steiger, R. 1995a, Space Sci. Rev., 72, 49

Geiss, J., Gloeckler, G., von Steiger, R., et al. 1995b, Science, 268, 1033

Gloeckler, G., Cain, J., Ipavich, F. M., et al. 1998, Space Sci. Rev., 86, 497

Gloeckler, G., Geiss, J., Balsiger, H., et al. 1992, A\&AS, 92, 267

Grevesse, N., \& Sauval, A. J. 1998, Space Sci. Rev., 85, 161

Jian, L., Russell, C. T., Luhmann, J. G., \& Skoug, R. M. 2006, Sol. Phys., 239, 337

Jian, L., Russell, C. T., \& Luhmann, J. G. 2011, Solar Phys., 274, 321

Kohl, J. L., Esser, R., Gardner, L. D., et al. 1995, Sol. Phys., 162, 313
McComas, D. J., Bame, S. J., Barker, P., et al. 1998, Space Sci. Rev., 86 563

McComas, D. J., Ebert, R. W., Elliott, H. A., et al. 2008, Geophys. Res. Lett., 35, L18103

Meyer, J. P. 1991, Adv. Space Res., 11, 269

Nolte, J. T., Krieger, A. S., Timothy, A. F., et al. 1976, Sol. Phys., 46, 303

Raymond, J. C., Kohl, J. L., Noci, G., et al. 1997, Sol. Phys., 175, 645

Richardson, I. G., \& Cane, H. V. 2010, Sol. Phys., 264, 189

Schwadron, N. A., Fisk, L. A., \& Zurbuchen, T. H. 1999, ApJ, 521, 859

Suess, S. T., Ko, Y.-K., von Steiger, R., \& Moore, R. L. 2009, J. Geophys. Res., 114, A04103

von Steiger, R., Geiss, J., \& Gloeckler, G. 1997, in Cosmic Winds and the Heliosphere, ed. J. R. Jokipii, C. P. Sonett, \& M. S. Giampapa (Tucson, AZ: Univ. Ariz. Press), 581

von Steiger, R, Schwadron, N. A., Fisk, L. A., et al. 2000, J. Geophys. Res., 105,27217

von Steiger, R., \& Zurbuchen, T. H. 2011, J. Geophys. Res., 116, A01105

von Steiger, R, Zurbuchen, T. H., \& McComas, D. J. 2010, Geophys. Res. Lett., 37, L22101

Wilhelm, K., Curdt, W., Marsch, E., et al. 1995, Sol. Phys., 162, 189

Wimmer-Schweingruber, R. F., von Steiger, R., \& Paerli, R. 1999, J. Geophys. Res., 104, 9933

Zhao, L., Zurbuchen, T. H., \& Fisk, L. A. 2009, Geophys. Res. Lett., 36, L14104

Zurbuchen, T. H. 2007, ARA\&A, 45, 297

Zurbuchen, T. H., Fisk, L. A., Gloeckler, G., \& von Steiger, R. 2002, Geophys. Res. Lett., 29, 1352

Zurbuchen, T. H., Hefti, S., Fisk, L. A., Gloeckler, G., \& Schwadron, N. A. 2000, J. Geophys. Res., 105, 18327

Zurbuchen, T. H., Hefti, S., Fisk, L. A., Gloeckler, G., \& von Steiger, R. 1999, Space Sci. Rev., 87, 353

Zurbuchen, T. H., \& Richardson, I. G. 2006, Space Sci. Rev., 123, 31 